\title{
Temporal Region, Surgical Anatomy: Keep it Simple!
}

\author{
Badr MI Abdulrauf, MD, FRCSC* \\ Section of Plastic Surgery, Department of Plastic Surgery, King Faisal Specialist Hospital and Research \\ Center, Jeddah, Saudi Arabia
}

\begin{abstract}
Due to the several layers of fasciae and their interchanging characteristics below and above the zygomatic arch, the temporal region anatomy has somewhat been unpleasant to comprehend and recall. The frontal branch of facial nerve is however the ultimate reason why it becomes important to study this area.

Many surgical specialties need to work on this region, some of the common procedures include Coronal approaches; Zygoma fracture reduction; Temporoparietal flap elevation; Face and brow lift.

We believe there is a need for clear and doubt free messages to be made in regard this topic. After an extensive literature search, we came up with few conclusions and key illustrations that we strongly believe are crucial to be remembered.

The rationale of following certain path of dissection in the temporal region, depending on the planned operation is explained. Eponyms used in literature for various structures have been discussed and clarified. The relationship of the frontal nerve to its surrounding fasciae within the zygomatic zone and Temporoparietal fascia is further explored. This review and guidelines are specifically been developed and recommended as an educational tool for in training surgical residents of concerned specialties.
\end{abstract}

Keywords

Temporal region, Frontal branch, Temporal branch, Facial nerve, Temporoparietal fascia, Deep temporal fascia, Anatomy

\section{Abbreviations}

TR: Temporal Region; SMAS: Superficial Musculoaponeurotic System; TPF: Temporoparietal Fascia; DTF: Deep Temporal Fascia; ZA: Zygomatic Arch; FN: Frontal Nerve

\section{Introduction}

The Temporal region (TR) is a common site for several surgical interventions and approaches, it has interesting anatomical details and it is a transition zone between the scalp; occiput; forehead and the face.

Surgical specialties that are frequently involved in this area include: Neurosurgery; Otorhinolaryngology; Head and Neck; Maxillofacial; Plastic aesthetic and Reconstructive surgery.

Historically, there are several excellent studies and papers by pioneer authors on ways to protect the frontal nerve (FN) branch of the facial nerve [1-5].

Stuzin, et al. came up with their famous study and were the first to describe location of the FN in relation to TPF, as being just underneath it when cephalad to the ZA. They advised the safe level of dissection would either be superficial to TPF or otherwise under the superficial layer of DTF within the superficial fat pad [1].

In fact, there are few anatomical variations in findings and opinions in regard trajectory of the nerve and ramifications.
However, fortunately there are less differences in opinions regarding the level at which the nerve takes its path [4].

What may make this region look often confusing is two folds: one, the terminology used for various layers, fasciae are inconsistent between the specialties and even among the same specialty. In fact, the nerve most relevant to this topic has been interchangeably referred as Temporal branch of the facial nerve in many references and often as the frontal branch of the facial nerve. There seems to be personal preferences to surgeons based on their basic anatomy backgrounds.

The other issue at this region is that, various surrounding

*Corresponding author: Dr. Badr MI Abdulrauf, MD, FRCSC, Program Director, Plastic surgery, Department of Surgery, King Faisal Specialist Hospital and Research Center, PO Box 40047, Jeddah 21499 , Saudi Arabia, Tel: (44)-7778029219

Accepted: September 26, 2020

Published online: September 28, 2020

Citation: BMI Abdulrauf (2020) Temporal Region, Surgical Anatomy: Keep it Simple!. Stud Anat Physiol 2(1):13-19 
structures or layers change their consistency, thicknesses and their order with the intervening components such as the fat pads, temporalis muscle and the zygomatic arch.

The main objectives of this report to discuss the level of dissection used for common functional and or aesthetic interventions and to clarify some of the terminologies used for various layers.

Beyond the Subcutaneous fat layer there is a general scheme of different layers of tissues in the face, unlike anywhere else in the body [4]:

1. Skin

2. Subcutaneous tissue

3. Superficial muscles, zygomaticus major and minor; orbicularis oculi; orbicularis oris and risorius.

4. Superficial musculoaponeurotic system (SMAS)- Platysma
-Temporoparietal fascia (TPF)- Frontalis- Galea aponeurotica- Occipitalis

5. Facial nerve plain (initially, it is under the parotid-masseteric fascia)

6. Deep Fascial system: Parotid-Masseteric fascia and the Deep temporal fascia (DTF)

\section{Deep muscles}

These structures are delineated as a Coronal cross section in the anterior temple at the junction with forehead, more emphasis and relative magnification was made to superficial layers to delineate the FN changing depth. (Figure 1). We have then illustrated the various layers from superficial to deep, in the face-head oblique view drawings (Figure 2 and Figure 3).

\section{Temporoparietal Fascial Flap}

TPF is a thin sheet of fascia with a temporal part anterior-

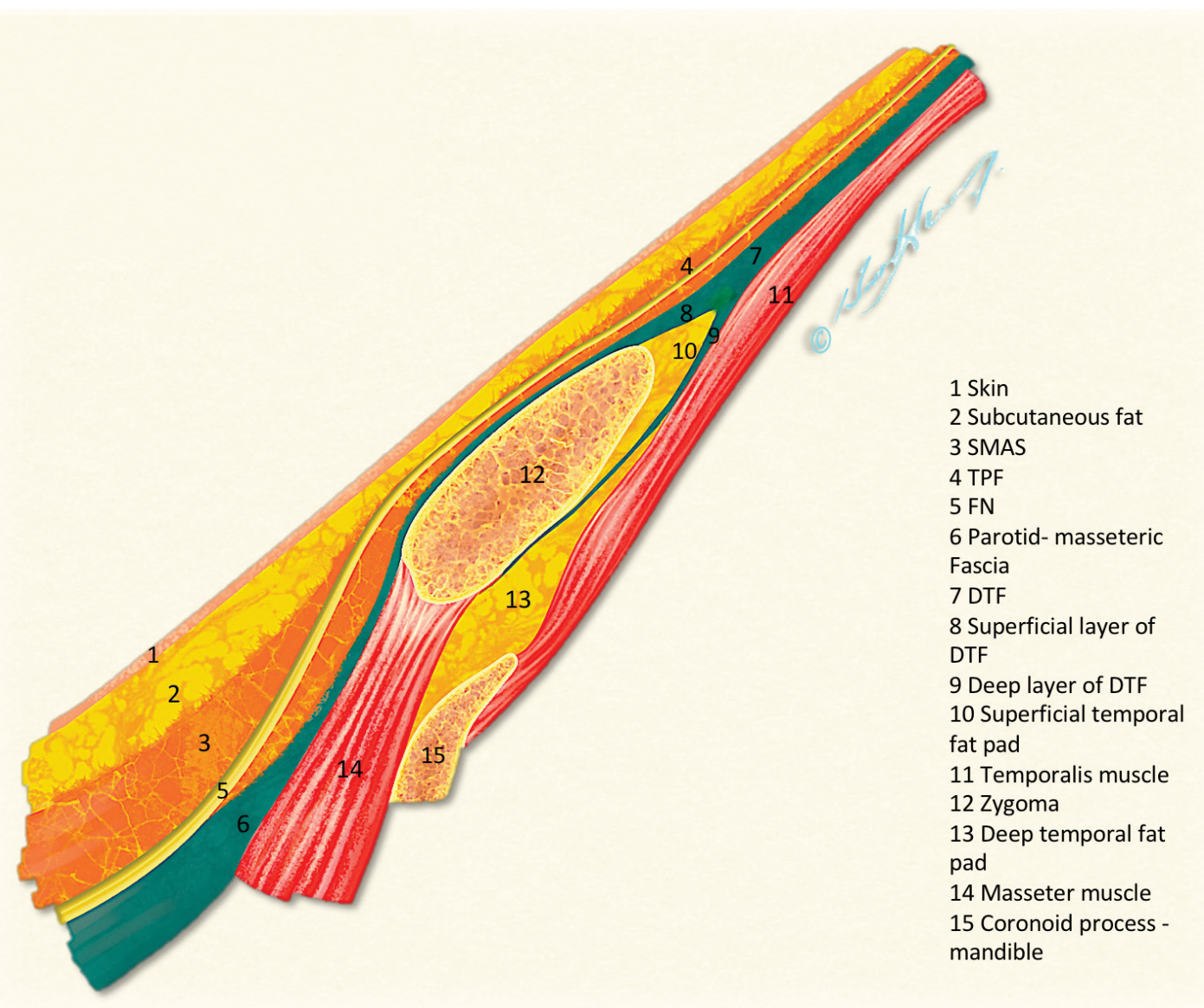

Figure 1: Superficial Coronal cross section to the right temporal region at junction with the forehead". The Frontal nerve (FN) or Temporal branch of facial nerve generally penetrates the SMAS about the level of zygomatic arch and becomes more superficial. Over the arch all layers are significantly thinned making the nerve especially vulnerable to injury. Cephalad to Zygomatic arch the FN is intimately adherent to TPF, which is in turn gradually been replaced by muscular fibers of frontalis. In other words, dissection in this area must be superficial to the Temporoparietal fascia (TPF) at the subcutaneous level or otherwise under the Deep temporal fascia (DTF) superficial layer then subperiosteally. This critical detail of the nerve's course is unfortunately not clearly shown in most of the reviewed literature's cross-sectional diagrams.

'We have magnified the superficial layers out of proportion to the deeper layers for the purpose of nerve's course illustration. The course of the nerve is extrapolated in this illustration based on extensive review and by imagining an overlap of multiple coronal cuts, to enable delineating the nerve's true and surgically relevant course. 


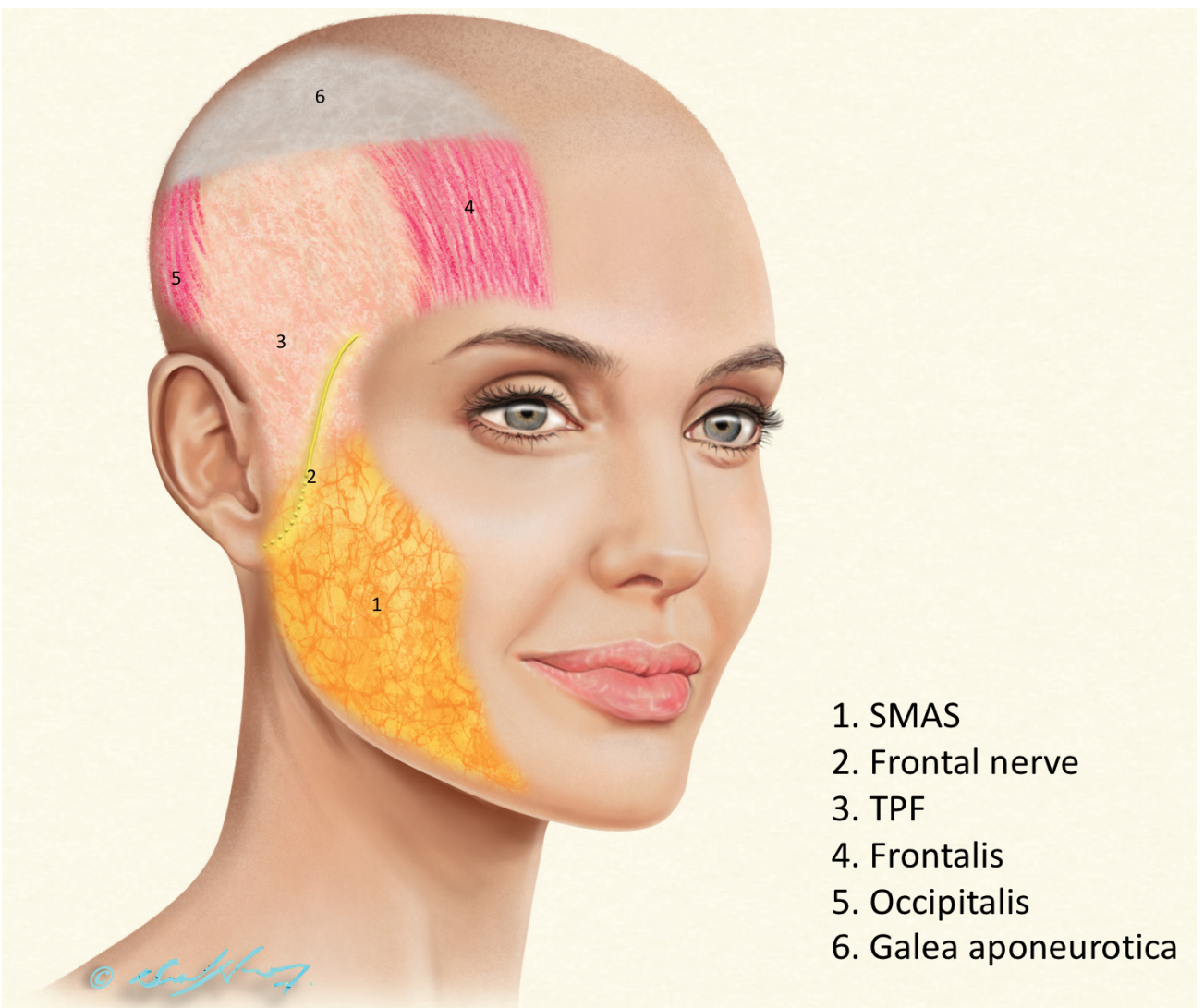

Figure 2: Author's depiction of the layer just underneath the subcutaneous fat on the face and head. This can be imagined as a mask with changing features in different areas. The SMAS over the cheek converts into the TPF as approaching the temporal area. The TPF is contiguous with the frontalis and occipitalis muscle fibers on forehead and occiput respectively. In between it is represented by the Galea aponeurotica.

The FN is shown with a glow, indicating its varying horizontal and longitudinal positions as well as its diverse level of branching and arborization patterns. However, for safe practice it is critical to consider the nerve is always in close adherence to SMAS and the TPF in this zone.

ly, a parietal part superiorly and a triangular part in between. It is the counterpart to, and contiguous with SMAS, frontalis, occipitalis and the galea (Figure 2).

The TPF flap is a relatively common and a very useful reconstructive tool for the ear or adjacent areas. It is based on the superficial temporal vessels which originate from the external carotid system. The arterial pulsation is usually easily palpable and a doppler can be used for accurate localization. The artery gives frontal, parietal and posterior branches.

Proximally in the preauricular area, the vascular pedicle is situated on surface of the TPF, however as going cephalad they gradually take more superficial position. At approximate level of $10 \mathrm{~cm}$ above the crus helix, the vessels penetrate the subdermal fat and eventually enter the subdermal plexus, generally at $12 \mathrm{~cm}$ above crus helix and that represents the limit of the fascial vascular domain [6].

The FN relationship to proper TPF in its most anterior portion is shown by most papers to be just underneath, but some have shown it to be within its substance (Figure 1) [7]. This fact makes it fair to state: the entire TPF tissue as a flap cannot be lifted off the nerve without causing an injury.

Injury to $\mathrm{FN}$ has been studied in relation to temporal arte- rial biopsy procedure and and in fact significant incidence has been published [8-10]. However, those are mostly reported by specialties with relatively less surgical experiences particularly in the TR.

Danger zone in relation to temporal artery biopsy has been investigated. An oblique line passing through 2 points, a $6.0 \mathrm{~cm}$ point from lateral canthus and $4.5 \mathrm{~cm}$ point from lateral margin of supraorbital rim has been described. Anterior or medial to this line is considered to be the danger zone. Posterior or lateral to this line is considered a safe zone for making an incision and taking a biopsy from the artery [11].

The branching patterns of the superficial temporal artery has been studied and has been used as a landmark to locate the FN for the sake of precaution in rhytidectomies. According to level of bifurcation and specifically the emerge of the frontal branch of the artery in relation to superior orbital rim, it was classified as a high location type (more common) and low location type. In the high location type, the FN courses deeply and antero- inferiorly to the artery. In the low location type, the FN twigs interweave with the artery [12].

Despite FN injury with TPF flap surgery has not been a significant issue as per plastic surgical literature (reported as 
a rare complication), we believe the above studies are quite relevant and beneficial to TPF flap surgery. In cases where a significant width of the TPF flap is needed and or considered, an extended attempt to involve the most anterior territory of TPF might potentially lead to FN injury.

\section{Face and Brow Lift}

Surgical facial rejuvenation surgery involves dissection, release of skin and deeper soft tissue layers and then suspending them in an appropriate vector.

It has been standard to extend the incision and dissection into the temporal region in classic rhytidectomies, in order to adequately address the tissue laxity and to enable lifting the midfacial ptotic tissues to a temporal direction. This means, there is a need to connect the dissection and pockets created on mid face to the one in the TR. Therefore, it is vital to understand the anatomy of the FN as it ascends from midface towards frontal region.

Most of the Coronal cross-sectional illustrations in literature did not reveal the FN full course in its supra-zygomatic portion. Instead it is commonly been shown as a round structure cross section lying just adherent or underneath the TPF, very much like a single Coronal cut on a CT scan. This can be noted on Stuzin's famous paper to begin with and then in so many other articles and books chapters [2-4,13-16].

Such drawings can be deceiving, since they give an impression the nerve is barely a single dimension structure passing in a single straight horizontal line.

In reality the nerve does course in an oblique manner from inferior-posterior to superior-anterior direction, this is what originally was shown in the study of Pitanguy and Ramos where nerve typically follows a trajectory from $0.5 \mathrm{~cm}$ below the tragus to $1.5 \mathrm{~cm}$ above the lateral brow (Figure 2) [1].

Owsley and Agarwal however in their cross-sectional diagrams have shown much more realistic representation of the $\mathrm{FN}$ and other branches as well in their intra-operative images [17].

Plication of the SMAS layer is a common maneuver as part of rhytidectomy, which simply means raising it as a triangular flap and tucking it in the peri auricular area.

Classical teaching has been, the FN as it ascends towards ZA it penetrates the SMAS and becomes superficial. SMAS plication would require transection of the SMAS vertically usually in preauricular area and horizontally. This horizontal incision to SMAS is theoretically at right angle to the axis of $\mathrm{FN}$, and should be done with extreme caution, typically and traditionally been advised to be well below the level of ZA to avoid the SMAS zone in which the nerve is changing its course to a superficial plain.

Over the zygomatic prominence all layers though present but become quite thin (Figure 1). Although some expert surgeons are capable to separate these layers safely, but in general it is advisable to keep the dissection just subcutaneous at this zone.
A Sub-SMAS pocket on the face and sub-TPF dissection pocket on the temple must never be connected, because otherwise the nerve will surely be lacerated while it is passing from underneath to within and above that layer [18].

In fact, the only safe level dissection that can be connected in face and temple would be either purely and barely subcutaneous or otherwise deep to the DTF, deep layer and subperiosteally in the face. These conclusions can be easily be made by a glimpse of the first illustration (Figure 1).

Theoretically speaking an exception to this rule would be a dissection quite far posterior (few millimeters within the preauricular area and its corresponding area parietally, which would not optimum in aesthetic procedures.

Agrawal, et al. have questioned whether the $\mathrm{FN}$ travels within the SMAS at any point, due to their clinical observation and on their cadaveric study, showing the FN does not sprout out of the SMAS to become superficial. As per their dissection and study, the FN crossed the zygomatic arch at deeper level than the SMAS. On that basis they justify making a horizontal incision to the SMAS at level of ZA or even above it contrary to what classic teaching has been [3].

In Coronal brow lift procedure, the dissection is initially under the TPF level until approximately $2 \mathrm{~cm}$ above the upper border of the ZA, the superficial layer of DTF is then penetrated and dissection continues within the superficial fat pad.

Endoscopic face lift is a procedure done in different planes to various regions, it is often combined with smaller open approaches and various authors of this concept have their preferences and combinations [19-21].

When brow lift is considered, the dissection is extended in sub-glaeal plane (superficial to periosteum) in the area medial to temporal crest. Forehead is usually elevated subperiosteally. Dissection in the TR is initially just above the DTF until it divides to superficial and deep layers at approximate level of the superior orbital rim. The superficial layer is then penetrated and dissection is preceded just above the fat pad without going within it, to keep the field bloodless. At the superior border of the ZA the periosteum is incised and a subperiosteal dissection is performed over the arch, which would provide a quite safe level in regard the FN.

The Sentinel vein is one of perforating vessels through various layers that are noted in the TR. This vein is usually located about half centimeter lateral to the frontozygomatic suture line. It was found to be a useful landmark during endoscopic brow lift [7].

Since the FN is always above (superior to) the vein, a zone of caution was identified based on the vein's pre and intra-operative localization. Upon vein's visualization intra-operatively the dissection must be slowed down and more cautious tissue handling is recommended.

\section{Reduction of Zygomatic Fracture}

This classic procedure was named after sir Gillies for treatment of an isolated ZA fracture [22]. The approach is through the DTF above the level of its split which is generally accepted to be approximately $2-3 \mathrm{~cm}$ above the $\mathrm{ZA}$. 


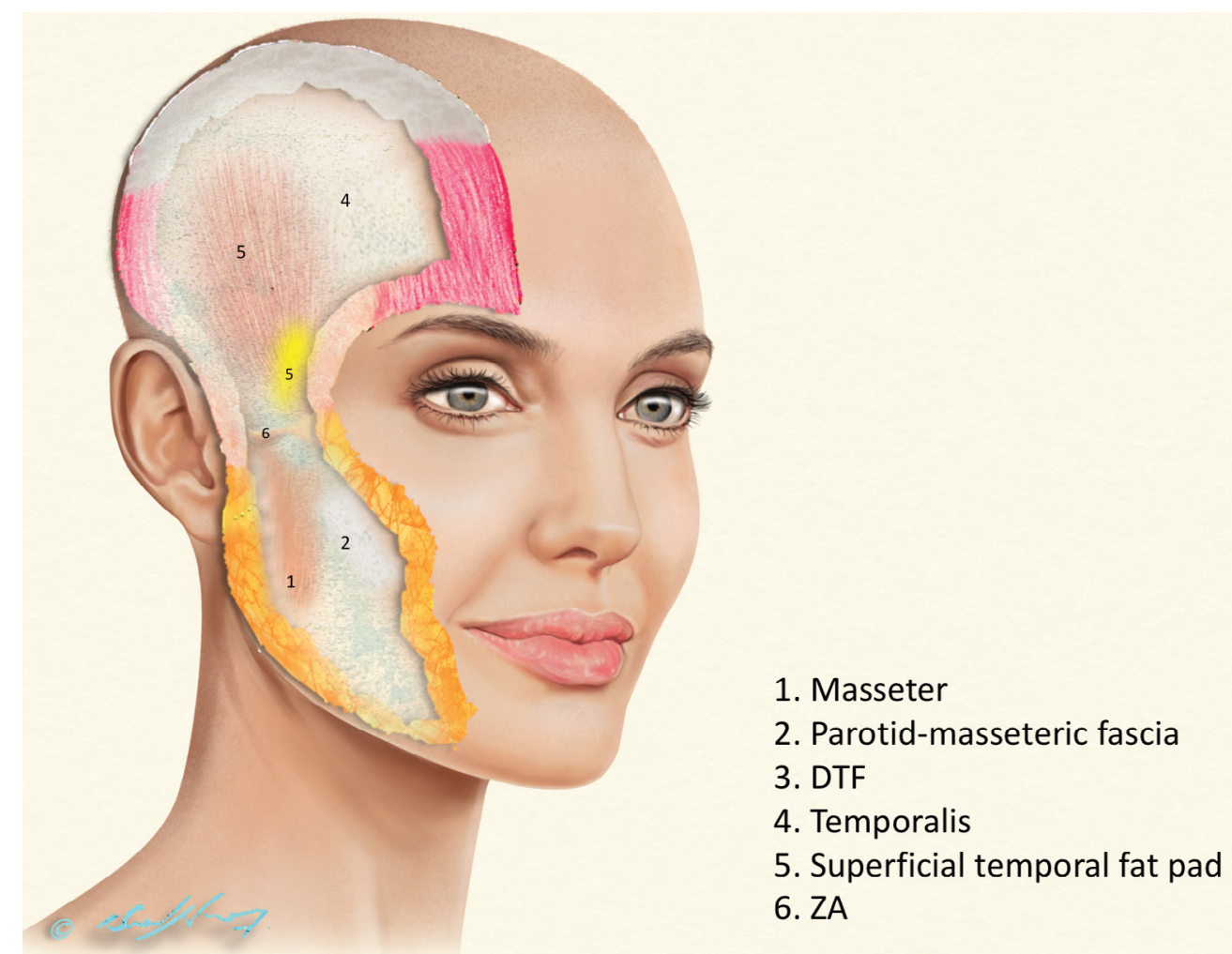

Figure 3: A window into the next layer. The Parotid-masseteric fascia in cheek region, which is the counterpart of the Deep temporal fascia (DTF) in the Temporal region, specifically its superficial layer. Reflections of temporalis, masseter muscles, zygomatic arch (ZA) and the superficial temporal fat pad can be observed underneath.

The Deep temporal fascia (DTF) has been occasionally referred in some papers as "the deep fascia". This well-defined fascia covers the outer surface of the temporalis muscle (Figure 3). Introducing an elevator deep to this layer ensures proper positioning of the elevator medial and under the ZA.

The superficial layer of DTF attaches to lateral border while the deep layer to the medial border of the ZA upper border. In between is the superficial fat pad which has a vein that needs to be avoided.

The level at which the DTF splits has been studied in details, different statistical values were reached. However, it was concluded that temporal incision is to be considered 5 $\mathrm{cm}$ from superior border of ZA or $2 \mathrm{~cm}$ superior to top of helix (This is important to make sure, in order not to be within the superficial temporal fat pad which may otherwise cause the vein rupture, hematoma and the inability to be effectively around the ZA for the reduction maneuver [23].

The presence of a deep layer of DTF has been questioned. In a prospective study while operating on 130 cases of zygomatico-facial fractures, the finding of absent identifiable fascial layer between the superficial temporal fat pad and the temporalis muscle was pointed out. The authors of this particular study believed in the non-existence of such a layer [24].

\section{Discussion}

The complication of an injury to facial nerve or one of its branches is equivalent to a major one such as pulmonary embolus following any major body contouring surgery or other, in terms of impact on patient and the surgeon.

When one has a complication, it is difficult to defend on basis of anatomic variation or on basis of unusual course of the nerve. It is therefore important to consider a danger zone rather than a trajectory for granted.

The percentage of reported facial nerve injury during rhytidectomy has a wide range of $1-20 \%$. However, world renowned face lift surgeons indicated in many meetings, there seems to be an underreporting to this complication, and it happens more frequent than thought. It should also be kept in mind, the rates in the literature are based on self-reported retrospective cases series by highly experienced surgeons. Although true transection of the nerve is uncommon, temporary compromise to the nerve and facial asymmetry is not rare.

Upon our critical review here to the major papers out of the numerous ones published in regard to anatomical studies and reviews to the FN in the TR, varying data were found in terms of distance measurements from stable structures.

This observation by itself is a clear indication that variability in relation to $\mathrm{FN}$ is very common. In addition, majority of the cadaveric studies on the facial nerve show all its branches except for the FN (Temporal branch), which has rarely been clearly demonstrated.

Therefore, it makes all the sense to be take a safety zone of caution. In other words, there is no typical location for this nerve that one can depend on. 
Diagnostic imaging with $\mathrm{CT}$ or MRI plays important role in diagnosing disease related processes affecting the facial nerve. This is mostly applicable to the main nerve's central (intra-cranial course) and extracranially within the parotid. CT has been useful for investigating traumatic injuries to the facial nerve with fractures of Temporal bone. MRI has been widely used to evaluate patients with schwannomas, hemangiomas or similar tumors affecting the facial nerve as well as parotid tumors. However, when it comes to iatrogenic injuries to branches such as the Temporal or Frontal (FN), these modalities have not been useful. Nor these investigations are used for preoperative delineation of the course for these branches [25].

In terms of terminology of various layers, many of the structures in the TR have been named differently.

Majority do agree, DTF splits into a superficial and deep leaflet. Hence, saying the term "superficial fascia" does not mean much, since it could indicate for many as the Temporoparietal fascia (TPF) or otherwise the superficial layer of the DTF.

Also, the term "Deep fascia" does not mean much since it could indicate to many as the proper DTF or otherwise the deep layer of the DTF.

Some papers have described and used the term "Innominate fascia" to the loose areolar tissue lying between the TPF and the DTF. They mentioned such a layer begins underneath the SMAS layer in region of the ZA, and the $\mathrm{FN}$ is being underneath it and it is protected. Hence in their hands, they feel safe to transect the SMAS at a higher level [3].

Davidge, et al. have done an extensive review of various eponyms in effort to standardize somewhat the terms used for various fasciae among specialties in this region [26]. Anatomy is a slippery science when it comes to recalling relationship of tissues and structures to each other, imaginary lines and measurements. This is why mnemonics are extensively been used.

Too many diagrams and anatomical relations to memorize, carry the risk of forgetting the vital information. One should be selective of what piece of anatomical knowledge or illustration to keep in mind [26-28].

\section{Conclusion}

1. Majority of the anatomic literature on FN are merely guidelines to help preventing its injury.

2. The FN runs at constant level in relation to fascial layers but with diverse trajectory zones, distances, branching level and arborizations.

3. Anatomical illustrations in cross section are in a single dimension, and can often be deceiving by giving incorrect sense in regard the depth of nerve and of its 3-dimensional relationships.

4. The antero-superior course of FN over ZA area and distally has unfortunately been neglected in majority of the Coronal cross-sectional diagrams in literature so far. The nerve travels in a semi-vertical direction in order to supply the frontalis, while lying within or attached to TPF.

5. The use of incomplete terms such as "the superficial fascia" or "the deep fascia" must be discouraged due the confusion and misunderstanding it may give rise to.

6. Three key diagrams have been proposed for simple understanding of layers in the TR, which in turn simplify the understanding for the rationale been recommended in regard the plain of dissection for common operations in this region. Students and concerned residents in training are encouraged to study them.

\section{References}

1. Pitanguy I, Ramos A (1966) The frontal branch of the facial nerve: The importance of its variations in face lifting Plast Reconstr Surg 38: 352.

2. Stuzin J, Wagstrom L, Kawamoto H, et al. (1989) Anatomy of the frontal branch of the facial nerve: The significance of the temporal fat pad. Plast Reconstr Surg 83: 265.

3. Agarwal C, Menedenhall S, Foreman K. et al. (2010) The course if the frontal branch of the facial nerve in relation to facial planes: An anatomic study. Plast Reconstr Surg 125: 532-537.

4. Stuzin B, Baker T, Gordon H (1993) The relationship of the superficial and deep fascias: Relevance to Rhytidectomy and aging. Plast Reconstr Surg 91: 441-449.

5. Roostaeian J, Rohrich R, Stuzin J (2014) Anatomical considerations to prevent facial nerve injury. Plast Reconstr Surg 135: 1318.

6. Byrd H (1998) Temporoparietal (Superficial temporal artery) fascial flap. Chapter in Grabb's encyclopedia of flaps. (2nd edn), Lippincott-Raven, Philadelphia.

7. Trinel F, Januszkiewicz J, Nahai F (1998) The Sentinel vein: An important reference point for surgery in the temporal region. Plast Reconstr Surg 101: 27-32.

8. Bhatti M, Goldstein M (2001) Facial nerve injury following superficial temporal artery biopsy. Dermatol Surg 27: 15-17.

9. Murchison A, Bilyk J (2012) Brow ptosis after temporal artery biopsy: Incidence and associations. Ophthalmology 119: 26372642.

10. Gunawardene A, Chant $H$ (2014) Facial nerve injury during temporal artery biopsy. Ann R Coll Surg Engl 96: 257-260.

11. Shin K, Shin H, Lee S (2018) Surgical anatomy of the superficial temporal artery to prevent facial nerve injury during arterial biopsy. Clin Anat 31: 608-613.

12. Lei T, Xu DC, Gao J, et. al. (2005) Using the frontal branch of the superficial temporal artery as a landmark for locating the temporal branch of the facial nerve during rhytidectomy: An anatomical study. Plast Reconstr Surg 116: 623-629.

13. Watanabe Koiici. Et al. (2016) Soft tissue of the scalp and temporal region. Anatomy for plastic surgery Theme.

14. Bongcheol K, Seungmin O, Wonsung J (2019) Anatomy for absorbable thread lifting. Springer.

15. Dong K, Young S, Woo S, et al. (2017) Reduction of isolated zygomatic arch fractures with Gillies approach. Korean J Oto H\&N Surg 61: 588-592.

16. Krayenbuhl N, Isolan G, Hafez A, et al. (2007) The relationship of 
the fronto-temporal branches of the facial nerve to the fascias of the temporal region: a literature review applied to practical anatomy dissection. Neurosur Rev 30: 8-15.

17. Owsley J, Agarwal C (2008) Safely navigating around the facial nerve in three dimensions. Clin Plast Surg 35: 469-477.

18. Giovanni B, Cerevallo M (2012) Midface and neck aesthetic plastic surgery, first edition. Acta Medica Edizioni SEE-FIRENZE.

19. Ramirez O (1995) Endoscopic forehead and face lift, step by step: Operative techniques in plastic and reconstructive surgery 2: $129-136$.

20. Mackay G, Nahai F (1995) The endoscopic forehead lift. Opersative techniques in plastic and reconstructive surgery 2: 137-144.

21. Byrd H, Salomon J (1998) Endoscopic midface rejuvenation. Operative techniques in plastic and reconstructive surgery 5: 138144.

22. Gillies HD, Kilner T, Stone D (1927) Fractures of the malar-zygomatic compound: With a description of a new $\mathrm{x}$-ray position. $\mathrm{Br}$ J Surg 14: 651-656.
23. Park J, Lee S, Ha T, et al. (2020) Location of the split of the deep temporal fascia when reducing a zygomatic arch fracture. J Plast Reconstr Aestht Surg 73: 1130-1134.

24. Li H, Li K, Jia W, et al. (2018) Does the deep layer of the deep temporal fascia really exist? J Oral Maxillofac Surg 76: 1824.e 1-1824.e7.

25. Gupta S, Mends F, Hagiwara M, et al. (2013) Imaging the facial nerve: A contemporary review. Radiology research and practice.

26. Davidge K, Van F, Agur A, et al. (2010) Naming the soft tissue layers of the temporoparietal region: Unifying anatomic terminology across surgical disciplines. Nerosurgery.

27. Smith CF, Border S (2019) The twelve cranial nerves of Christmas: Mnemonics, rhyme, and anatomy - seeing the lighter side. Anat Sci Educ 12: 673-677.

28. Abdulrauf BMI (2020) A mnemonic for neck triangles. Anat Physiol 10: 323. 